\title{
Investigation of the Metal Cations Adsorption Selectivity Using Nanocavities-Rich Polyamine-Cross-Linked PMVEAMA
}

\author{
Mateusz Pawlaczyk $^{1}$, Grzegorz Schroeder ${ }^{1}$ \\ ${ }^{1}$ Faculty of Chemistry, Adam Mickiewicz University in Poznań \\ Uniwersytetu Poznańskiego 8, Poznań, Poland \\ mateusz.pawlaczyk@amu.edu.pl; schroede@amu.edu.pl
}

\begin{abstract}
Water contamination with toxic metal ions is one of the major problems of environmental pollution, caused by intensified economic development. Therefore, efficient sorptive systems capable of binding metal cations are of great interest. Herein, we present the synthesis of materials containing nanocavities created by the cross-linking of poly(methyl vinyl ether-alt-maleic anhydride) with four structurally different polyamines: tris(2-aminoethyl)amine (TREN), piperazine, triethylenetetramine (TETA), and 4,7,10-trioxa-1,13-tridecanediamine (TRI-OXA). The easiness of the synthetic protocol and the biocompatibility of bare polymer indicate the convenience of the proposed adsorbents. The materials were subjected to adsorption of $\mathrm{Al}(\mathrm{III}), \mathrm{Mn}$ (II), $\mathrm{Hg}$ (II), and Cd(II) ions from their binary, ternary, and quaternary systems. The ions' adsorption percentages were established using XRF analysis, indicating the dependence of the materials' adsorption ability on the cross-linking agent used. Such findings are strictly related to the size of the polyamine used, determining the distances between subsequent polymer chains, and thus the size of internal nanocavities formed.
\end{abstract}

Keywords: cross-linked polymers, nanocavity, toxic metal ions, adsorption, XRF, ions adsorption selectivity

\section{Introduction}

Polymers are one of the most broadly created, studied, and implemented materials since they exhibit easily tunable chemical and physical properties. The final polymers' properties, which indicate their applicability, are dependent especially on the choice of functional monomers, but also on the reaction conditions (temperature, sonochemical factor, photo- or thermal-induction), the choice of initiator, and the chosen medium. Moreover, synthetic or biosourced polymeric chains may be used in the cross-linking process, which concerns the interconnection of subsequent polymeric chains with a multifunctional - at least bi-functional - molecule, called a cross-linker. The resulting polymer network exhibits new properties in reference to bare polymer, e.g. increased mechanical properties and gained elasticity, but also swelling capability, owing to created pores between the polymeric chains. The process of cross-linking is very broadly applied, especially in the synthesis of elastomers, such as tires, rubbers, gaskets, and sealing devices, using several types of agents as diols, diamines, or dithiols [1]. Nevertheless, such phenomenon occurs also in the natural environment and human organisms, exemplary in proteins self-stabilization via disulfide bridges or cross-linking of cancer DNA or RNA strands using sulfur yperite as a non-selective treatment of various cancer diseases [2].

The mentioned properties' improvements by the cross-linking process have jointly prompted its implementation in designing various applied polymeric materials. For example, 3D cross-linked polymer net of poly(ethylene glycol) dimethyl acrylate (PEGDA) and ethoxylated trimethylpropane triacrylate (ETPTA) has been proposed as solid electrolyte interphase - a matrix for lithium ions storage derived from dual-salt LiTFSI/LiPF 6 , finding application as highly efficient and reusable batteries ingredient [3]. Moreover, two different reagents: triptycene and phenylmethylsilicone were subjected to their cross-linking using formaldehyde dimethyl acetal (FDA), leading to highly absorptive material dedicated to $\mathrm{CO}_{2}$, volatile organic compounds (VOCs), such as $\mathrm{CHCl}_{3}, \mathrm{CH}_{2} \mathrm{Cl}_{2}$, cyclohexane, and synthetic dyes. The systems have been proved to adsorb synthetic dyes extremely fast and swelling organic solvents even several-fold times the sample weight [4-5]. The cross-linking process may also lead to a product of enhanced thermal stability, e.g. polyacrylamide crosslinked with hydroquinone and hexamethylenetetramine, strengthened with silica nanoparticles [6], but also maybe be 
used

a synthesis of "smart" polymers, such as self-healing or healing systems, based on diarylbibenzofuranone cross-linked with hexamethylene diisocyanate and triethanolamine. The healing and self-healing properties were afforded by the equilibrium state of the linkages in the polymer net. The material showed very good recoverability, i.e. complete recovery after 12 hours at $50^{\circ} \mathrm{C}$, dependent on the temperature of the environment [7].

Significant attention has also been paid to materials based on natural or bio-derived domains. Such materials are designed mostly as potential adsorbents dedicated to various aqueous contaminants, such as toxic metal ions, synthetic organic dyes, or phenol-derivatives. The literature presents several scientific reports, which aimed at the description of adsorptive properties of materials based on cross-linked chitosan or cellulose and $\beta$-cyclodextrin ( $\beta$-CD). For instance, chitosan cross-linked with poly(alginic acid) $/ N, N$ '-methylene-bis-acrylamide, and poly(methacrylamide) was proved for satisfactory removal of $\mathrm{Cr}(\mathrm{VI})$ and $\mathrm{Pb}$ (II) ions from aqueous solutions, respectively [8-9], while cellulose cross-linked with epichlorohydrin showed good performance in adsorption of $p$-nitrophenol and single component carboxylates such as 2-dicyclohexylacetic acid or adamantane carboxylic acid [10]. Moreover, a dual-biopolymeric material classified as Schiff base, consisting of chitosan and dialdehyde cellulose, was investigated for adsorption of model protein - bovine serum albumin (BSA) [11]. Interestingly, chitosan-based beads cross-linked with sodium tripolyphosphate or glutaraldehyde have been also determined as efficient adsorbents dedicated to structurally different mycotoxins, involving adsorption of carcinogenic, nephrotoxic, and hepatotoxic aflatoxin B1, fumonisin B1, and zearalenone [12]. When it comes to the application of $\beta$-cyclodextrin-derivatives in water purification, the cross-linked materials are able to attach adsorbate molecules through various interactions. For example, $\beta-C D$ cross-linked with citric acid has been proved as a highly efficient material scavenging simultaneously different types of molecules, which were Bisphenol A - an endocrinedisrupting chemical, Methylene Blue - an organic dye, and $\mathrm{Cu}(\mathrm{II})$ ions. The adsorption of the dye and the metal ions was afforded by incorporation of citric acid domains, interacting with the analytes through electrostatic interactions and chelation, while the adsorption of the hydrophobic contaminant was afforded by host-guest supramolecular inclusion to the interior of macrocyclic saccharide's residues, which leads to the non-competitive adsorption of different analytes [13]. Such host-guest inclusion was also studied for $\beta-C D$ cross-linked with glutaraldehyde, maleic anhydride, ethylenediaminetetraacetic acid (EDTA), or FDA, as adsorptive materials towards various organic dyes and phenolic compounds [14-17]. Efficient adsorbents based on cross-linked polymeric products dedicated to toxic metal ions were obtained using rectorite/chitosan or $\beta$-CD/tetrafluoro-terephthalonitrile systems [18-19], while chloromethylated polystyrene cross-linked using dichloroethane and co-polymer of glycidylmethacrylate and divinylbenzene post-crosslinked with dichloroethane were studied as adsorbents towards aniline and salicylic acid, respectively [20-21].

The recent study aimed at cross-linking of poly(methyl vinyl ether-alt-maleic anhydride) (PMVEAMA) with structurally different polyamines, which were tris(2-aminoethyl)amine, piperazine, triethylenetetramine, and 4,7,10-trioxa1,13-tridecanediamine, leading to materials full of nanocavities between subsequent cross-linking domains. The choice of the polymeric support was driven by its proven biocompatibility and mucoadhesiveness [22], as well as by the ease of its functionalization through the opening of the anhydride domains under the nucleophilic attack of free amine groups. Such materials may exhibit differences in the pore size, caused by the length of the polyamine used, which may significantly change the affinity of materials towards specific ionic species [23-24]. Herein, the obtained materials were tested for the adsorption of toxic metal ions commonly polluting natural water reservoirs. The experiments included adsorption of cations of different size and valency - $\mathrm{Al}(\mathrm{III}), \mathrm{Mn}(\mathrm{II}), \mathrm{Hg}(\mathrm{II})$, and $\mathrm{Cd}(\mathrm{II})$ - from their binary, ternary, and quaternary systems, which helped for indicating the dependence between the nanocavities obtained during the cross-linking processes and the affinity for entrapment of cations of a particular size. 


\section{Materials and methods}

\subsection{Chemicals}

All the reagents used for the synthesis of the materials, i.e. poly(methyl vinyl ether-alt-maleic anhydride) (average $M_{w} \sim 216000$, average $\left.M_{n} \sim 80000\right)$, tri(2-aminoethyl)amine (96\%), piperazine (99\%), triethylenetetramine (70\%), and 4,7,10-trioxa-1,13-tridecanediamine (97\%), and the salts used for adsorption experiments, i.e. aluminum perchlorate nonahydrate (98\%), manganese(II) perchlorate hydrate (99\%), mercury(II) perchlorate tetrahydrate $(99.99 \%)$,

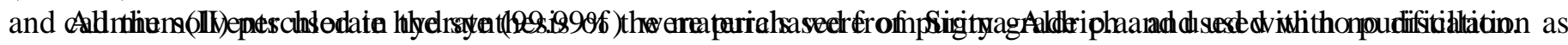
a purification step. The used DMF was purchased from Eurochem BGD (Tarnów, Poland), and dried over molecular sieves $4 \AA$ for 24 hours before its use for the synthesis. $\mathrm{Et}_{2} \mathrm{O}$ was supplied by POCH (Gliwice, Poland), and toluene was supplied by Stanlab (Lublin, Poland).

\subsection{Instruments}

The synthesized materials were characterized by Fourier-Transform Infrared spectroscopic (FT-IR) analysis using Bruker $^{\circledR}$ IFS 66v/S spectrometer. The samples were introduced into $\mathrm{KBr}$ pellets and analyzed in the range of 400-4000 $\mathrm{cm}^{-1}$. Thermogravimetric (TG) assays were performed using Setsys 1200 Setram $^{\circledR}$ analyzer, operating in a heating mode with temperature step $5^{\circ} \mathrm{C} \mathrm{min}^{-1}$ in the temperature range $25-1000^{\circ} \mathrm{C}$. The $\mathrm{X}$-Ray Fluorescence $(\mathrm{XRF})$ analysis was performed using MiniPal 2 PANalytical $^{\circledR}$ instrument. All the material-ions complexes were analyzed under given conditions: air atmosphere, time $=300 \mathrm{~s}$, filter $=$ Kapton, excitation energy $=25 \mathrm{kV}$, and auto-set intensity of

the X-ray source (tube with a rhodium target) varying between 11 and $97 \mu \mathrm{A}$. The instrument's software allowed for establishing the percentage of contents of each metal in the samples. All the measurements were done in duplicate in order to avoid any artifacts.

\subsection{Synthesis of Cross-Linked Poly (Methyl Vinyl Ether-Alt-Maleic Anhydride)}

For the synthesis of cross-linked PMVEAMA particles, a general procedure was as follows: into a three-necked round-bottom flask, equipped with a magnetic stirrer and thermometer, $6.4 \mathrm{mmol}$ of particular amine was added and dissolved in $40 \mathrm{~mL}$ of anhydrous DMF. The solution was purged with $\mathrm{N}_{2}$ and heated to $100^{\circ} \mathrm{C}$. The amounts of amines were: $0.998 \mathrm{~mL}$ of tris(2-aminoethyl)amine (TREN) $\left(146.24 \mathrm{~g} \mathrm{~mol}^{-1} ; 0.977 \mathrm{~g} \mathrm{~mL}^{-1}\right), 0.56 \mathrm{~g}$ of piperazine $\left(86.14 \mathrm{~g} \mathrm{~mol}^{-}\right.$ $\left.{ }^{1}\right), 1.362 \mathrm{~mL}$ of triethylenetetramine (TETA) $\left(146.24 \mathrm{~g} \mathrm{~mol}^{-1} ; 0.982 \mathrm{~g} \mathrm{~mL}^{-1}\right)$, and $1.446 \mathrm{~mL}$ of 4,7,10-trioxa-1,13tridecanediamine

(TRI-OXA) (220.31 $\left.\mathrm{g} \mathrm{mol}^{-1} ; 1.005 \mathrm{~g} \mathrm{~mL}^{-1}\right)$. Then, to the heated amine solution, a solution of $2.0 \mathrm{~g}$ of PMVEAMA in $100 \mathrm{~mL}$ of anhydrous DMF was added dropwise. During the addition of the polymer solution, the stirring was vigorous in order to avoid the formation of a gel and maintained for 5 hours. Afterwards, the mixture was calmly stirred at $70^{\circ} \mathrm{C}$ under a nitrogen atmosphere for 16 hours. After that time, the mixture was cooled to room temperature and $100 \mathrm{~mL}$ of cooled $\mathrm{Et}_{2} \mathrm{O}$ was added. The precipitates were centrifuged and washed with $100 \mathrm{~mL}$ of cooled $\mathrm{Et}_{2} \mathrm{O} 2$ times. Then, the materials were dried under vacuum at $45^{\circ} \mathrm{C}$ for 8 hours, yielding dark green-blue p-TREN, pale pink p-piperazine, brown p-TETA, and dark yellow p-TRI-OXA materials. The cross-linked materials were obtained with yields ranging between 92 and $98 \%$.

\subsection{Adsorption of the Toxic Metals on the Cross-Linked Polymeric Materials}

The adsorption experiments involved the interaction of the samples of the cross-linked materials with various toxic metal ions solutions in their binary, ternary, and quaternary systems. The general procedure involved mixing 100 $\mathrm{mg}$ samples of each material with $20 \mathrm{~mL}$ of an aqueous solution of cationic systems with the final concentration of each cation equal to $0.01 \mathrm{mM}$. The binary cationic systems were: $\mathrm{Al} / \mathrm{Mn}, \mathrm{Al} / \mathrm{Hg}, \mathrm{Mn} / \mathrm{Hg}$, and $\mathrm{Cd} / \mathrm{Hg}$; the ternary systems: $\mathrm{Al} / \mathrm{Mn} / \mathrm{Hg}, \mathrm{Al} / \mathrm{Cd} / \mathrm{Hg}, \mathrm{Mn} / \mathrm{Cd} / \mathrm{Hg}$, and $\mathrm{Al} / \mathrm{Mn} / \mathrm{Cd}$; and the quaternary system: $\mathrm{Al} / \mathrm{Mn} / \mathrm{Cd} / \mathrm{Hg}$. The adsorption was conducted for 24 hours at room temperature, followed by centrifugation of the material-cations complexes and their double washing 
with distilled water. The materials with the adsorbed metal ions were dried under vacuum at room temperature for 24 hours.

The dried samples were subjected to XRF analysis

\section{Results and discussion}

\subsection{Synthesis and Characterization of the Cross-Linked Polymeric Materials}

The chosen easy-accessible, biocompatible, and easy-modifiable polymeric material PMVEAMA has been considered for the cross-linking process using structurally different polyamines. The easiness in the process is afforded by the high affinity of the maleic anhydride domains in the polymer to be opened under the attack of a nucleophilic agent (in this case an amine group). Nevertheless, the processes were handled at elevated temperature, approximately $100^{\circ} \mathrm{C}$, affording full-opening of the polymer's anhydride rings. The polyamine character of the reagents used led to the linking of two subsequent polymer chains through amide bonds (Figure 1), yielding in nano-sized cavities.

a)

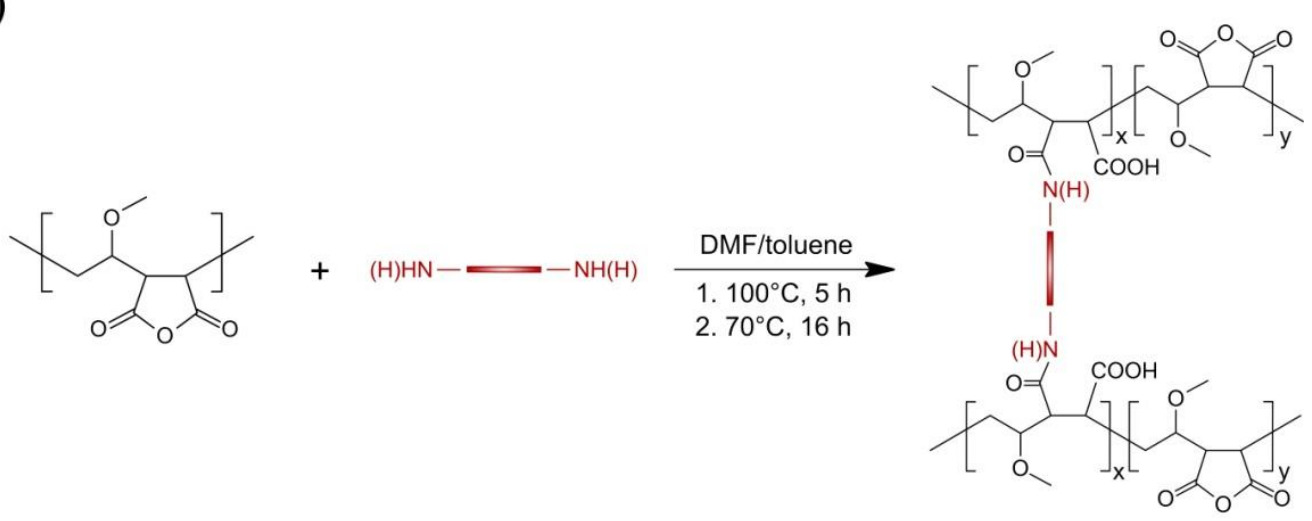

b)

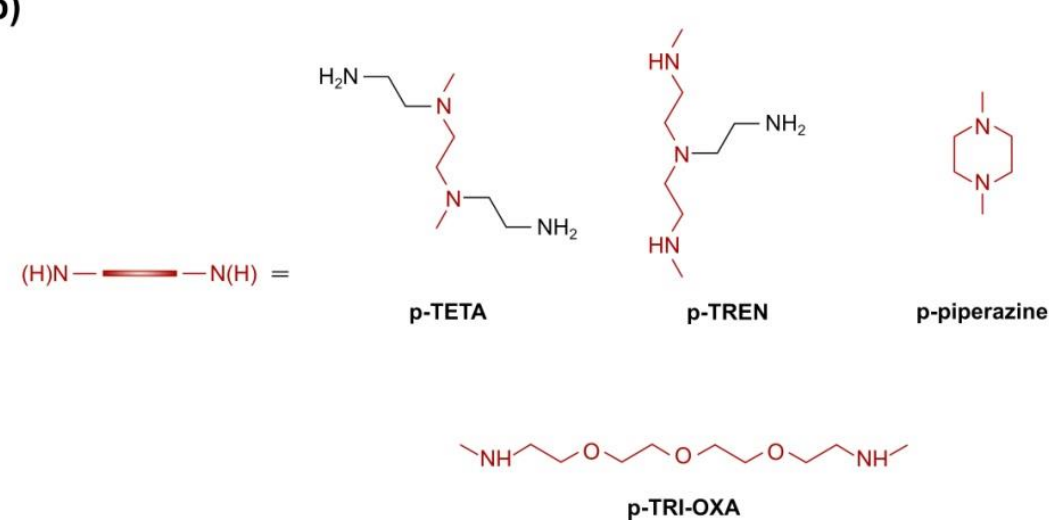

Fig. 1: a) The synthetic route for the obtaining of the cross-linked polymers; b) the structure of the polyamines used.

The obtained materials were characterized by two analytical techniques: FT-IR and TG measurements. The FT-IR spectra of bare polymer and the cross-linking products are collected in Figure 2. The spectrum of PMVEAMA exhibits several distinct signals corresponding to 5-membered anhydride ring domains, which are the stretching of $\mathrm{C}=\mathrm{O}$ groups of anhydride at 1780 and $1858 \mathrm{~cm}^{-1}$, and the stretching of $\mathrm{C}-\mathrm{O}-\mathrm{C}$ anhydride structural residues at $927 \mathrm{~cm}^{-1}$. The success of PMVEAMA cross-linking with all the chosen amines is proved by a disappearance of the given signals in the materials' spectra, which proves the full-opening of maleic anhydride rings. Moreover, the spectra of the cross-linked materials exhibit common signals corresponding to the formation of amide groups and the incorporation of amine groups. 
These signals are 1700 and $\sim 1662 \mathrm{~cm}^{-1}$ - the stretching of $\mathrm{C}=\mathrm{O}$ groups of formed carboxylic and $2^{\circ}$ - or $3^{\circ}$-amide groups, respectively; $\sim 1439 \mathrm{~cm}^{-1}-$ the stretching of $\mathrm{C}-\mathrm{N}$ domains of amide and/or amine groups incorporated to PMVEAMA platform; or $\sim 1403 \mathrm{~cm}^{-1}$ - the stretching of $\mathrm{O}-\mathrm{H}$ group of formed carboxylic groups. Also, all the spectra exhibit a broad band centered at around $3430 \mathrm{~cm}^{-1}$, which is related to a single or overlapped stretching of $\mathrm{N}-\mathrm{H}$ and $\mathrm{O}-\mathrm{H}$, the

signals at approximately 2935 and/or $2851 \mathrm{~cm}^{-1}$, corresponding to the stretching of $-\mathrm{CH}_{2}-$ domains, and also a sharp signal at $1095 \mathrm{~cm}^{-1}$ assigned as the stretching of $\mathrm{C}-\mathrm{O}$ ether groups [25]. Moreover, thermogravimetric analysis of the crosslinked materials has been performed, which curves are presented in Figure 2. Each spectrum exhibits a first decomposition step at around $120^{\circ} \mathrm{C}$, which is attributed to the loss of the solvent molecules trapped within the materials' cavities. Although in the spectra of p-TREN, p-piperazine, and p-TETA there is a single step at approximately $200^{\circ} \mathrm{C} \quad$ connected with the oxidation of pendant amine residues not inter-connecting the polymeric chains, the main broad step between 230 and $650^{\circ} \mathrm{C}$ prove the cross-linking of the chains with used polyamine agents (otherwise, the spectra would have shown separated signals originating in the oxidation of the amine and the polymer).
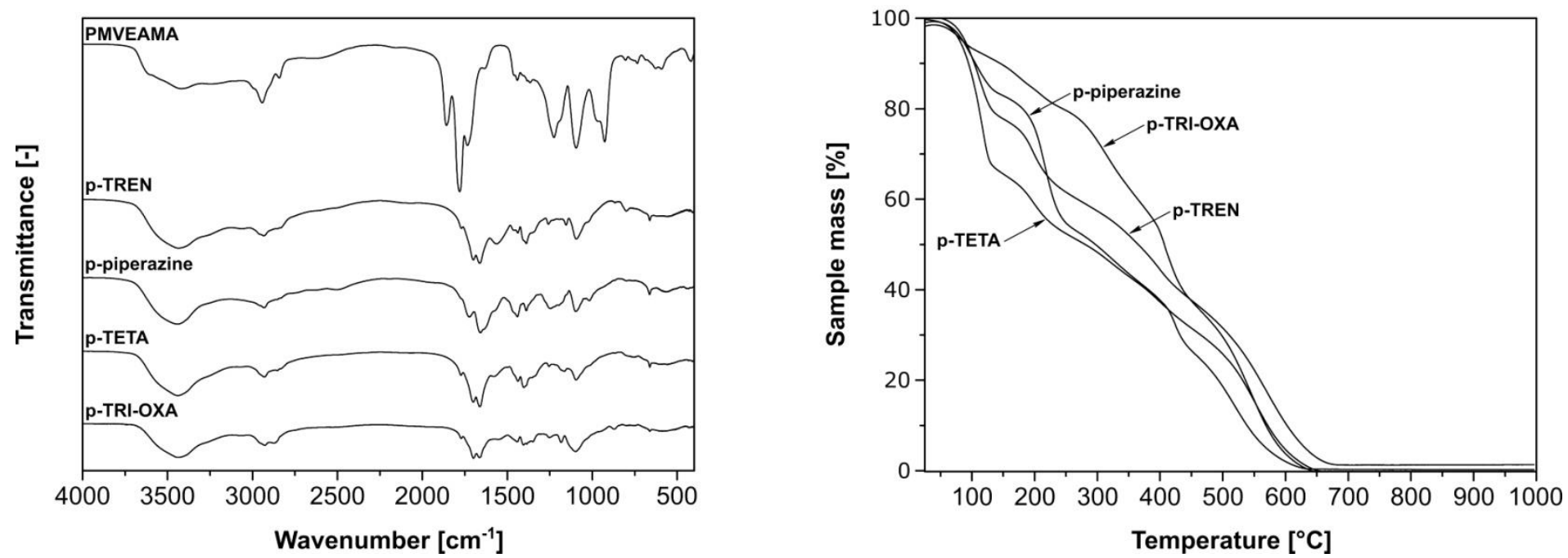

Fig. 2: The FT-IR spectra of the bare polymer and the cross-linked materials (left) and TG curves obtained for the materials (right).

\subsection{Adsorption of the Toxic Metals on the PMVEAMA-Based Materials and Evaluation of the Selectivity}

The obtained cross-linked polymeric materials with multiple internal nanocavities were dedicated to the adsorption of widely polluting toxic metal ions: $\mathrm{Al}(\mathrm{III}), \mathrm{Mn}(\mathrm{II}), \mathrm{Hg}(\mathrm{II})$, and $\mathrm{Cd}$ (II). The choice of a particular amine as a cross-linking agent is expected to define the distance between two subsequent polymeric chains and thus influencing the size of created nano-sized internal cavities between them. The chosen metal cations differ in their ionic radii, which may have an impact on their ability to diffuse into the cavities' interior. The mean ionic radii calculated by Shanon et al. are $0.468,0.795,1.080$, and $1.007 \AA$ for $\mathrm{Al}(\mathrm{III}), \mathrm{Mn}(\mathrm{II}), \mathrm{Hg}(\mathrm{II})$, and Cd(II), respectively [26]. Therefore, all the cross-linking products were tested for the adsorption of the metal cations from their various systems: binary, ternary and quaternary ones. Percentages of the metal cations adsorbed on each material from the particular aqueous system were established using XRF analysis of material-ions complexes. The contact time was set at 24 hours, in order to reach an equilibrium state, needed for reliable measurements. The raw experimental data presenting specific percentages values collected in Table 1, while the graphical representation of the selectivity of the adsorbent towards specific cations is presented in Figure 3. 
Table 1: The percentages of the metal cations adsorbed from various ion systems on the cross-linked polymeric materials.

\begin{tabular}{c|cccc|cccc|ccccc|cccc}
\hline Material & \multicolumn{4}{|c|}{ p-TREN } & \multicolumn{4}{c|}{ p-piperazine } & \multicolumn{4}{c|}{ p-TETA } & \multicolumn{3}{c}{ p-TRI-OXA } \\
\hline Cations & Al & Hg & Cd & Mn & Al & Hg & Cd & Mn & Al & Hg & Cd & Mn & Al & Hg & Cd & Mn \\
& 68.0 & & & 32.0 & 65.0 & & & 35.0 & 67.6 & & & 32.4 & 73.2 & & & 26.8 \\
& 99.6 & 0.4 & & & 60.9 & 39.1 & & & 99.8 & 0.2 & & & 96.7 & 3.3 & & \\
& & 63.8 & & 36.2 & & 79.2 & & 20.8 & & 64.0 & & 36.0 & & 72.4 & & 27.6 \\
\% of the ion & 41.0 & 36.5 & & 22.5 & 38.0 & 46.8 & & 15.2 & 35.6 & 44.0 & & 20.4 & 33.0 & 51.1 & & 15.9 \\
in the & & 100 & 0 & & & 67.1 & 32.9 & & & 100 & 0 & & & 53.0 & 47.0 & \\
material-ions & 99.2 & 0.8 & 0 & & 59.0 & 13.1 & 27.9 & & 36.0 & 39.0 & 25.0 & & 49.0 & 23.2 & 27.8 & \\
complex & & 45.0 & 0 & 55.0 & & 42.6 & 36.0 & 21.4 & & 22.0 & 0 & 78.0 & & 51.4 & 29.0 & 19.6 \\
& 66.0 & & 24.0 & 10.0 & 36.7 & & 31.3 & 32.0 & 53.3 & & 29.0 & 17.7 & 48.0 & & 28.0 & 24.0 \\
& 61.0 & 14.0 & 20.0 & 5.0 & 44.0 & 11.9 & 34.0 & 10.1 & 65.0 & 11.0 & 19.0 & 5.0 & 27.0 & 39.4 & 21.0 & 12.6 \\
\hline
\end{tabular}

a)

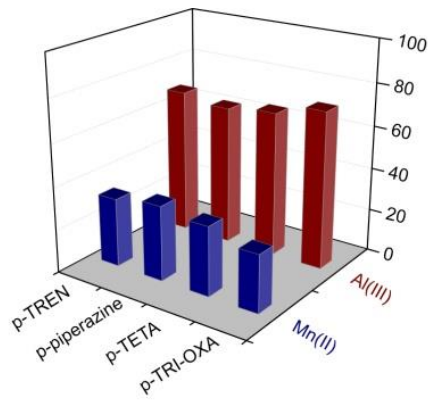

b)

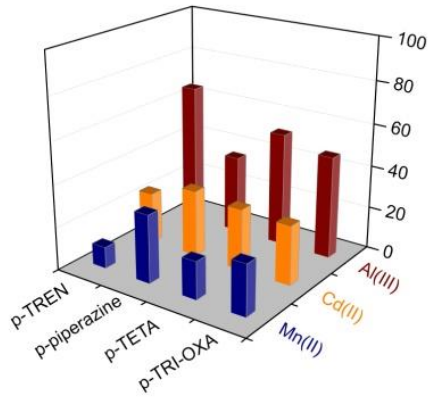

c)

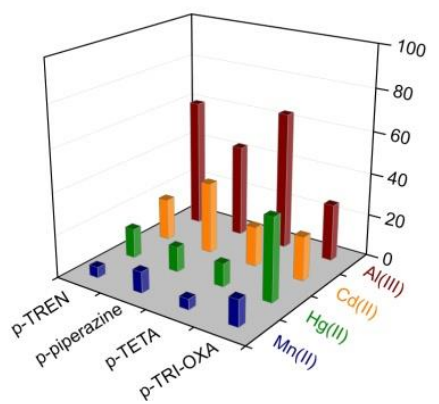

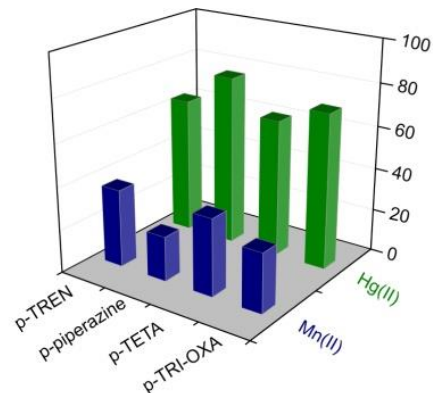
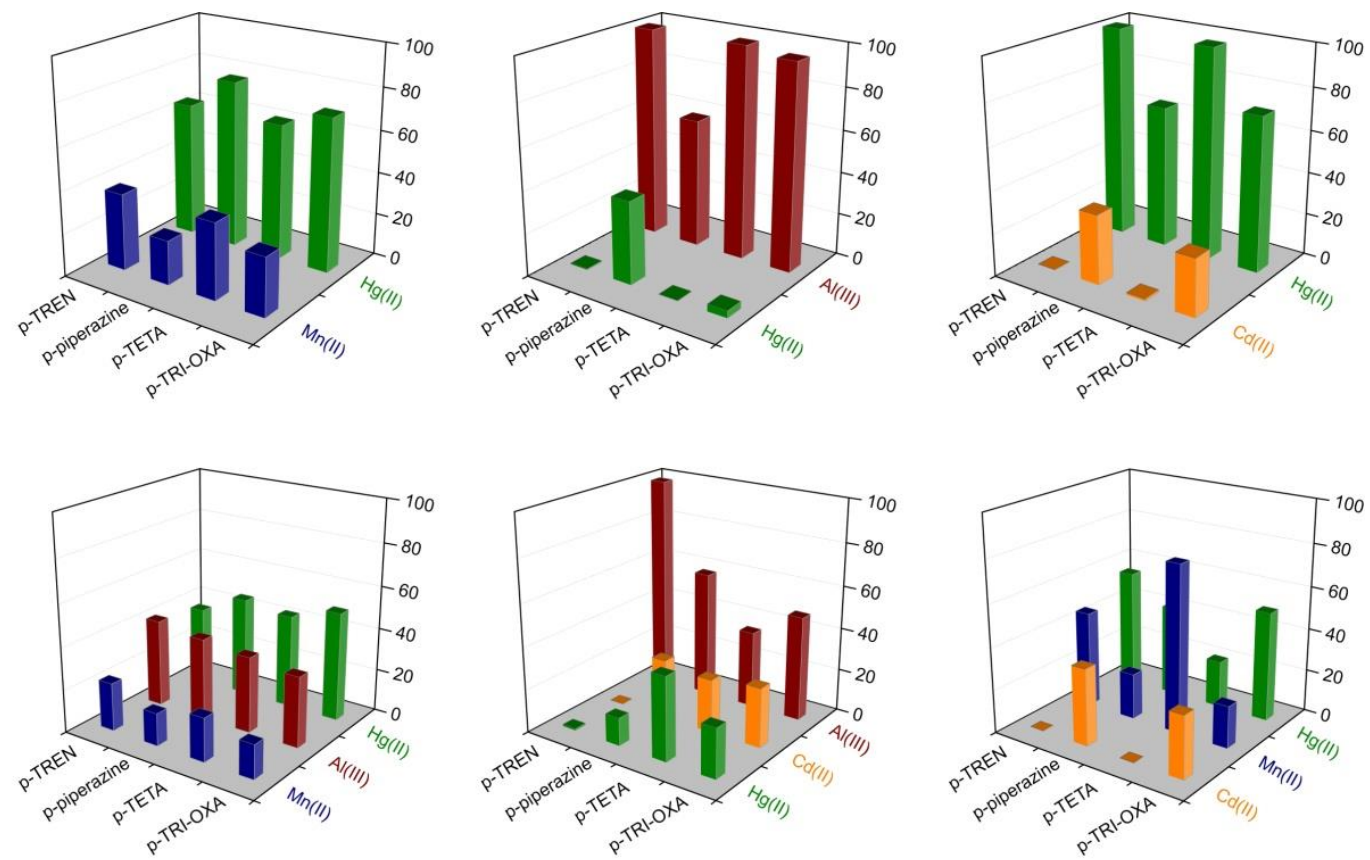

Fig. 3: The graphical representation of the ions adsorption selectivity from their various systems: a) binary, b) ternary, c) quaternary. 
In all the experiments involving the adsorption of $\mathrm{Al}$ (III) ions, their adsorbed percentage is predominant among all the ions adsorbed, which is strictly connected to the smallest size among all the ions studied, allowing for the easiest diffusion into materials pores and cavities obtained during the cross-linking step. The binary, ternary and quaternary Al-containing systems showed their adsorption percentage varying between 60.9 and 99.8, 33.0 and 99.2, and 27 and $65 \%$, respectively. On the other hand, $\mathrm{Hg}(\mathrm{II})$ ions as the largest ionic species exhibit high affinity towards the polymeric materials, especially when in the binary systems with $\mathrm{Mn}$ (II) or Cd(II) ions. It may be a result of their diffusion into entrapping pores and nanocavities, with subsequent hindering of the binding sites, allowing only for the diffusion of $\mathrm{Al}(\mathrm{III})$ ions, as the smallest ones. In general, the lowest sorption effectiveness was found for Cd(II) and $\mathrm{Mn}$ (II) ions. When it comes to the selectivity of the materials, an interesting phenomenon was observed for $\mathrm{p}$ piperazine material, which among all the cross-linked materials showed the highest percentage of $\mathrm{Cd}(\mathrm{II})$ ions $\begin{array}{llllll}\text { adsorption } & \text { of } & 27.9 & \text { to } & 36.0 \% & \text { Piperazine }\end{array}$ the only cyclic diamine used, which structural features may either improve or hinder the sorption of particular ions.

The experimental data may then indicate the most satisfactory fitting of Cd(II) ions within amine-ring nanocavity among all the other ions. Moreover, the materials cross-linked with TREN and TETA undoubtedly show decreased affinity towards "large" cations: $\mathrm{Hg}(\mathrm{II})$ and $\mathrm{Cd}(\mathrm{II})$. Such observation may be a result of the steric hindrance appearing upon

the cross-linking with branched polyamines, decreasing the size of created internal nanocavities. Also, pendant $-\mathrm{NH}_{2}$ groups of these agents may interact with formed $-\mathrm{COOH}$ groups via electrostatic interactions, leading to repulsive interaction with metal cations, especially those of large size (cadmium, mercury ions).

\section{Conclusion}

The recent studies aimed at the synthesis of amine-cross-linked PMVEAMA chains containing internal nanosized cavities. The use of the structurally different polyamines (branched and/or length-varying amines) contributed to the formation of nanocavities of different sizes between polymer chains. The materials were characterized with FT-IR and thermogravimetric analysis and then investigated for their adsorption capability towards chosen toxic metal ions: $\mathrm{Al}(\mathrm{III}), \mathrm{Mn}(\mathrm{II}), \mathrm{Hg}(\mathrm{II})$, and $\mathrm{Cd}(\mathrm{II})$. The adsorption processes were performed in various cationic systems, including the materials' contact with mixtures of two, three, or four metal cations simultaneously. The differences in the materials' structure and the size of the ions led to interesting results, e.g. the highest affinity of the materials to the smallest $\mathrm{Al}(\mathrm{III})$ ions, the highest affinity of p-piperazine material to $\mathrm{Cd}(\mathrm{II})$ ions among all the obtained materials. Such results may be also influenced by the use of cyclic piperazine ring as a cross-linker, as well as the electrostatic interactions between pendant amine groups of the cross-linking agent and carboxylic domains in polymeric chains formed after maleic anhydride

ring-opening.

The presented materials were obtained with high yields using a simple synthetic protocol, which increases their applicability as convenient toxic metal scavengers. Based on the current experimental data, the materials may be used not only as metal ions binding systems for purification of aqueous samples but also as pre-concentration tools allowing for further analysis of material-metal ions complexes.

\section{Acknowledgments}

The work was supported by grant no. POWR.03.02.00-00-I026/16 co-financed by the European Union through the European Social Fund under the Operational Program Knowledge Education Development. 


\section{References}

[1] D. Kuckling, A. Doering, F. Krahl, K.-F. Arndt, "8.15 - Stimuli-Responsive Polymer Systems", in Polymer Science: A Comprehensive Reference, Newnes, 2012, vol. 8, pp. 377-413.

[2] O. I. Orlova, E. I. Savel'eva, G. V. Karakashev, "Methods of Determination of Sulfur Yperite-DNA Adducts", J. Anal. Chem., vol. 72, no. 3, pp. 256-263, 2017.

[3] W. Fan, N.W. Li, X. Zhang, S. Zhao, R. Cao, Y. Yin, Y. Xing, J. Wang, Y.G. Guo, C. Li, “A Dual-Salt Gel Polymer Electrolyte with 3D Cross-Linked Polymer Network for Dendrite-Free Lithium Metal Batteries", Adv. Sci., vol. 5, no. 9, pp. 1800559-180067, 2018.

[4] C. Zhang, P.C. Zhu, L. Tan, J.M. Liu, B. Tan, X.L. Yang, H.B. Xu, “Triptycene-Based Hyper-Cross-Linked Polymer Sponge for Gas Storage and Water Treatment", Macromolecules, vol. 48, no. 23, pp. 8509-8514, 2015.

[5] L. Ding, H. Gao, F. Xie, W. Li, H. Bai, L. Li, "Porosity-Enhanced Polymers from Hyper-Cross-Linked Polymer Precursors", Macromolecules, vol. 50, no. 3, pp. 956-962, 2017.

[6] Y. Liu, C. Dai, K. Wang, C. Zou, M. Gao, Y. Fang, M. Zhao, Y. Wu, Q. You, "Study on a Novel Cross-Linked Polymer Gel Strengthened with Silica Nanoparticles”, Energy Fuels, vol. 31, no. 9, pp. 9152-9161, 2017.

[7] K. Imato, A. Takahara, H. Otsuka, "Self-Healing of a Cross-Linked Polymer with Dynamic Covalent Linkages at Mild Temperature and Evaluation at Macroscopic and Molecular Levels", Macromolecules, vol. 48, no. 16, pp. 5632$5639,2015$.

[8] G. Sharma, M. Naushad, A.H. Al-Muhtaseb, A. Kumar, M.R. Khan, S. Kalia, Shweta, M. Bala, A. Sharma, "Fabrication and characterization of chitosan-crosslinked-poly(alginic acid) nanohydrogel for adsorptive removal of Cr(VI) metal ion from aqueous medium", Int. J. Biol. Macromol., vol. 95, pp. 484-493, 2017.

[9] Z.A. Sutirman, M.M. Sanagi, K.J.A. Karim, W.A.W. Ibrahim, "Preparation of methacrylamide-functionalized crosslinked chitosan by free radical polymerization for the removal of lead ions", Carbohydr. Polym., vol. 151, pp. 1091-1099, 2016.

[10] I.A. Udoetoka, R.M. Dimmicka, L.D. Wilson, J.V. Headley, "Adsorption properties of cross-linked celluloseepichlorohydrin polymers in aqueous solution", Carbohydr. Polym., vol. 136, pp. 329-340, 2016.

[11] U.J. Kim, Y.R. Lee, T.H. Kang, J.W. Choi, S. Kimura, M. Wada, "Protein adsorption of dialdehyde cellulosecrosslinked chitosan with high amino group contents", Carbohydr. Polym., vol. 163, pp. 34-42, 2017.

[12] Z. Zhao, N. Liu, L. Yang, J. Wang, S. Song, D. Nie, X. Yang, J. Hou, A. Wu, "Cross-linked chitosan polymers as generic adsorbents for simultaneous adsorption of multiple mycotoxins", Food Control, vol. 57, pp. 362-369, 2015.

[13] W. Huang, Y. Hu, Y. Li, Y. Zhou, D. Niu, Z. Lei, Z. Zhang, "Citric acid-crosslinked $\beta$-cyclodextrin for simultaneous removal of bisphenol A, methylene blue and copper: The roles of cavity and surface functional groups", J. Taiwan Inst. Chem. Eng., vol. 82, pp. 189-197, 2018.

[14] J.A. Pellicer, M.I. Rodríguez-López, M.I. Fortea, C. Lucas-Abellán, M.T. Mercader-Ros, S. López-Miranda, V.M. Gómez-López, P. Semeraro, P. Cosma, P. Fini, E. Franco, M. Ferrándiz, E. Pérez, M. Ferrándiz, E. NúñezDelicado, J.A. Gabaldón, "Adsorption Properties of $\beta$ - and Hydroxypropyl- $\beta$-Cyclodextrins Cross-Linked with Epichlorohydrin in Aqueous Solution. A Sustainable Recycling Strategy in Textile Dyeing Process", Polymers, vol. 11, no. 2, pp. 252-272, 2019.

[15] Y. Jiang, B. Liu, J. Xu, K. Pan, H. Hou, J. Hu, J. Yang, "Cross-linked chitosan/ $\beta$-cyclodextrin composite for selective removal of methyl orange: Adsorption performance and mechanism", Carbohydr. Polym., vol. 182, pp. 106-114, 2018.

[16] F. Zhao, E. Repo, D. Yin, Y. Meng, S. Jafari, M. Sillanpää, "EDTA-Cross-Linked $\beta$-Cyclodextrin: An Environmentally Friendly Bifunctional Adsorbent for Simultaneous Adsorption of Metals and Cationic Dyes", Environ. Sci. Technol., vol. 49, no. 17, 10570-10580, 2015.

[17] H. Li, B. Meng, S.H. Chai, H. Liu, S. Dai, "Hyper-crosslinked $\beta$-cyclodextrin porous polymer: an adsorptionfacilitated molecular catalyst support for transformation of water-soluble aromatic molecules", Chem. Sci., vol. 7, no. 2, 905-909, 2016. 
[18] Y. Chen, Q. Zhang, X. Guo, Y. Peng, H. Xiao, X. Chen, J. Luo, “Adsorption of Cd(II), Cu(II) and Ni(II) ions by cross-linking chitosan/rectorite nano-hybrid composite microspheres", Carbohydr. Polym., vol. 130, pp. 333-343, 2015.

[19] J. He, Y. Li, C. Wang, K. Zhang, D. Lin, L. Kong, J. Liu, "Rapid adsorption of Pb, Cu and Cd from aqueous solutions by $\beta$-cyclodextrin polymers", Appl. Surf. Sci., vol. 426, pp. 29-39, 2017.

[20] W. Kuang, Y.N. Liu, J. Huang, "Phenol-modified hyper-cross-linked resins with almost all micro/mesopores and their adsorption to aniline", J. Colloid Interf. Sci., vol. 487, pp. 31-37, 2017.

[21] X. Ling, H. Li, H. Zha, C. He, J. Huang, "Polar-modified post-cross-linked polystyrene and its adsorption towards salicylic acid from aqueous solution", Chem. Eng. J., vol. 286, pp. 400-407, 2016.

[22] J.M. Irache, M. Huici, M. Konecny, S. Espuelas, M.A. Campanero, P. Arbos, "Bioadhesive Properties of Gantrez Nanoparticles", Molecules, vol. 10, no. 1, pp. 126-145, 2005.

[23] M. Pawlaczyk, G. Schroeder, "Adsorption studies of Cu (II) ions on dendrimer-grafted silica-based materials" J. Mol. Liq., vol. 281, pp. 176-185, 2019.

[24] M. Pawlaczyk, G. Schroeder, "Efficient Removal of Ni(II) and Co(II) Ions from Aqueous Solutions Using Silicabased Hybrid Materials Functionalized with PAMAM Dendrimers" Solvent Extr. Ion Exch., vol. 38, no. 5, pp. 496$521,2020$.

[25] G. Socrates, "1. Introduction", in Infrared and Raman Characteristic Group Frequencies, $3^{\text {rd }}$ Edition, Chichester, 2001, pp. 1-49.

[26] D.C. Ghosh, R. Biswas, "Theoretical Calculation of Absolute Radii of Atoms and Ions. Part 2. The Ionic Radii", Int. J. Mol. Sci., vol. 4, no. 6, pp. 379-407, 2003. 